\title{
The prevalence of hyperhidrosis in patients with spinal cord injuries and an evaluation of the effect of dextropropoxyphene hydrochloride in therapy
}

\author{
L S Andersen MD,${ }^{1} \mathrm{~F}$ Biering-Sørensen MD PhD,${ }^{2} \mathrm{P} \mathrm{G}$ Müller MD ${ }^{2}$ I L.Jensen MD ${ }^{1}$ \\ B Aggerbeck DVM ${ }^{3}$
}

${ }^{1}$ The Paraplegic Function, Department of Rheumatology, Viborg Hospital, Viborg, Denmark, ${ }^{2}$ Centre for Spinal Cord Injured, Department TH, Rigshospitalet, University Hospital, Copenhagen, ${ }^{3}$ Benzon Pharma A/S, Copenhagen.

The prevalence of annoying hyperhidrosis $(\mathrm{HH})$ in patients with spinal cord traumatic lesions was investigated by a questionnaire. A total of 192 patients were sent the questionnaire, 154 patients answered, and 41 patients reported annoying sweating. Of these 41 patients, 13 had a somatic underlying cause and 28 indicated having annoying $\mathrm{HH}$ without a contributing somatic cause.

Twenty-five patients with spinal cord injury (SCI) were included in a doubleblind, randomized, placebo-controlled, crossover trial using dextropropoxyphene hydrochloride (DP) in a slow release form (Abalgin Retard ${ }^{\circledR} 150 \mathrm{mg}$ Benzon Pharma A/S, Copenhagen) twice a day, for the treatment of annoying $\mathrm{HH}$. Nineteen patients with lesions between C4 and L4 completed the study. Eight found the active drug to be so effective that they wanted to continue the treatment while 3 preferred placebo. Six patients dropped out, 5 due to adverse effects. There was a trend towards an effect on sweating in daytime $(p=0.08-0.14)$. Given that the patients had a preference, which 15 of 19 had, the true frequency of patients preferring active treatment ranged from 32 to $84 \%$ (95\% exact confidence limits). For those with SCI above T6 level the limits ranged from 40 to $97 \%$.

We conclude that in spite of the lack of statistically significant effect, it seems worthwhile to try DP for annoying $\mathrm{HH}$, especially in patients with lesions above T6 level.

Key words: spinal cord injury; hyperhidrosis; reflex sweating; prevalence; dextropropoxyphene hydrochloride.

\section{Introduction}

Profuse hyperhidrosis $(\mathrm{HH})$ is a condition that is seen in spinal cord injured (SCI) patients. Its aetiology is not completely understood, but it is generally referred to as a dysfunction of the autonomic nervous system and the thermoregulation due to the spinal cord lesion. ${ }^{1}$

Excessive and annoying $\mathrm{HH}$ has presented a therapeutic problem, because the medical management is either unsuccessful or has unacceptable adverse effects. For example anticholinergic drugs may cause impairment of voiding, and adrenergic and ganglionic blocking drugs may cause postural hypotension.

Tashjian and Richter ${ }^{2}$ reported 2 cases of $\mathrm{HH}$ in SCI whom they treated successfully with dextropropoxyphene hydrochloride (DP). The authors recommended a further clinical trial on the subject.

Treatment of $\mathrm{HH}$ aims primarily at inhibiting activity in the sympathetic cholinergic innervated eccrine sweat glands. The mechanism of action of DP in this matter is purely speculative. Since DP acts as an agonist at opioid receptors, it may have the same effects on the autonomic nervous system as morphine. Animal studies with morphine have provided evidence to support the concept that opioids may act as weak ganglionic blocking agents, ${ }^{3}$ and morphine seems to attenuate the sympathetic efferent discharge at the central nervous system level in man. ${ }^{4}$

We therefore carried out a double-blind, 
randomized, placebo controlled, cross-over trial to investigate the therapeutic effect of DP on annoying $\mathrm{HH}$ in SCI. Furthermore, to determine the prevalence of annoying $\mathrm{HH}$, a questionnaire study was carried out at one of the two centres involved (The Paraplegic Function in Viborg).

\section{Methods}

Questionnaire study of the prevalence of annoying $\mathrm{HH}$ among SCI patients

Using a questionnaire, 192 patients with SCI regularly controlled at the Paraplegic Function in Viborg were asked if they had previously experienced or currently had annoying $\mathrm{HH}$, and, if so, whether they would like to receive medical treatment for it.

\section{Study on the effect of DP}

All SCI patients with annoying $\mathrm{HH}$ who were regularly controlled at one of the 2 centres for SCI in Denmark were offered participation in the study. Patients were included if their $\mathrm{HH}$ had started after their SCI, and if they had had annoying $\mathrm{HH}$ at least once a week for one month or more.

Patients were excluded if (1) there was an obviously treatable somatic cause of the $\mathrm{HH}$, for instance distention/inflammation of abdominal organs, infections, pressure sores, climacteric sweating etc; (2) $\mathrm{HH}$ occurred only in relation to autonomic hyperreflexia; (3) $\mathrm{HH}$ was present only in relation to bladder or bowel distention or emptying; (4) they were taking phenytoin, carbamazepine, pyridostigmine or DP; (5) they were allergic to DP; (6) they were found unable to cooperate or (7) they were alcoholics.

According to the Helsinki Declaration II, all patients were incubated after informed consent. Approval from the local scientific ethics committees was obtained. The patients were informed of the possible adverse effects of DP.

Before the trial all the patients were asked how often (in a week), and when (day and/or night) they had $\mathrm{HH}$ and how annoying it was (very annoying/annoying).

After randomisation the patients were allocated to either DP or placebo in the first trial period of 14 days, and this was followed immediately by cross-over to the second trial period of 14 days. DP was given in a slow release form (Abalgin Retard ${ }^{\circledR}$ capsules $150 \mathrm{mg}$, Benzon Pharma A/S Copenhagen) twice a day to obtain a steady plasma concentration. Every morning and evening the patients recorded on a visual analogue scale how annoying they felt the sweat secretion had been during the preceding 12 hours. Furthermore, after each trial period, the patients were asked whether they had felt any change in sweat secretion during the night or the day. After the second period patients were asked if any of the trial periods were found to have beneficial effects on the sweating, and if they would like to continue either of the treatments. In addition, possible adverse effects were noted. Medical treatment and dosage changes during the study periods were recorded.

\section{Statistical methods}

The primary effect variable was the patient preference. A two-sided Prescott test ${ }^{5}$ was used to investigate whether preferences could be referred to either the period or the treatment. As no statistically significant difference was found, the exact confidence limits for the $p$ value were determined (binomial distribution). A 2 split-plot variance analysis was used on the median visual analogue scale profiles for period and treatment effect. A Prescott test was performed on adverse effects and drop outs. A Mann Whitney rank sum test was used to investigate whether the changes in sweating after the first and the second trial period were significantly different. Fisher's exact two-sided test was used in analysis of $2 \times 2$ contingency tables. A significance level of $5 \%$ was chosen.

\section{Results}

\section{Prevalence of annoying $\mathrm{HH}$ in SCI}

A total of 192 patients were given the questionnaire; 154 (80.2\%) answered (see Table I). Eighty-seven patients had not experienced any sweating problems. Eleven had previous but not current problems: 6 reported that they had had very annoying 
HH 2-8 years after the spinal cord lesion; one reported a good effect of acupuncture; 2 that the sweating had disappeared after an operation for prostatic hypertrophy and one after having started intermittent catheterisation; and one reported that the $\mathrm{HH}$ had abruptly disappeared after a short period of treatment with epidural morphine.

Current $\mathrm{HH}$ problems were reported by 56 patients. Fifteen of the 56 answered that the sweating did not bother them or that they regarded it as a useful body signal, eg indicating too tight shoes, not being well seated, a too tightly fixed condom catheter, constipation or a full bladder. Thus, 41 patients reported annoying $\mathrm{HH}$. In 13 of these a somatic cause was found to be possibly contributing: infections, dyspepsia, climacteric sweating, autonomic hyperreflexia, palindromic rheumatism, or sweating only in conjunction with micturition or defaecation. No contributing somatic cause could be found in 28 patients $(14.6 \%)$ who indicated having annoying $\mathrm{HH}$.

\section{Study of the effect of DP}

During a 2 year period a total of 25 patients (see Table II) were included from the 2 centres who are covering the rehabilitation function for all the SCI patients in Denmark. Nineteen patients completed the study, while 6 patients dropped out, due to unacceptable adverse effects during DP treatment $(n=5)$ or fear of such $(n=1)$. The patients who completed the study were 14 men and 5 women, age 20 to 78 years (median 36). The time since the lesion ranged from 2 months to 41 years (median 5 years). The spinal cord lesions were located between $\mathrm{C} 4$ and L4. Eight patients had cervical lesions and all of them reported sweating above the level of the lesion. Fourteen had thoracic lesions and 7 of these had lesions above or at T6. Three patients had lumbar lesions. In most cases the sweating was located either in all the dermatomes proximal or distal to the level of the lesion. In some cases the sweating was not so well defined, but covered only parts of the corresponding areas (marked with $*$ in Table II). In patients with lesion levels below $\mathrm{T} 9$ the sweating tended to be more diffusely located (ie face, head, breast, axillae, trunk, or back). In 14 patients $\mathrm{HH}$ was most annoying during the day, and 17 patients had sweating at least once a day. There were no differences in the frequency of sweating between the patients with cervical or thoracic lesions. $\mathrm{HH}$ was significantly $(p=0.05)$ more annoying among cervical SCI compared with the lower lesions. There were no significant differences between treatment sequences, age, or gender, nor between patients from the 2 centres. There were no significant differences in the effects of DP among patients with proximal versus distal sweating or complete versus incomplete lesions.

Fifteen of the 19 patients had a preference. Nine preferred the drug and 6 preferred placebo. There was no statistically significant difference between the 2 treatments. The $95 \%$ confidence interval for the true proportion of patients who preferred DP, given that the patient had a preference, was $32-84 \%$. Considering patients with SCI above or at the level of T6 only (the lowest

Table I Prevalence of hyperhidrosis

\begin{tabular}{|c|c|c|c|}
\hline & No. of patients & $\begin{array}{l}\text { Percen } \\
\text { asked }\end{array}$ & $\begin{array}{l}\text { f patients } \\
\text { responding }\end{array}$ \\
\hline Asked & 192 & \multirow{3}{*}{$80.2 \%$} & \multirow{3}{*}{$100 \%$} \\
\hline Responding & 154 & & \\
\hline No $\mathrm{HH}$ at all & 87 & & \\
\hline Previously but no current $\mathrm{HH}$ & 11 & \multirow{3}{*}{$29.0 \%$} & \multirow{3}{*}{$36.3 \%$} \\
\hline Current HH & 56 & & \\
\hline Not annoying $\mathrm{HH}$ & 15 & & \\
\hline Annoying $\mathrm{HH}$ & 41 & \multirow[t]{2}{*}{$21.4 \%$} & \multirow[t]{2}{*}{$26.6 \%$} \\
\hline Contributing somatic cause & 13 & & \\
\hline No contributing somatic cause & 28 & $14.6 \%$ & $18.1 \%$ \\
\hline
\end{tabular}


Table II Patients participating in the dextropropoxyphene hydrochloride (DP) study with the localisation and severity of their hyperhidrosis $(\mathrm{HH})$ given together with their preference in the study

\begin{tabular}{|c|c|c|c|c|c|c|}
\hline $\begin{array}{l}\text { Patient } \\
\text { no. }\end{array}$ & $\begin{array}{l}\text { Gender } \\
(\mathrm{M} / \mathrm{F})\end{array}$ & Lesion level & $\begin{array}{l}\text { HH localisation } \\
(\mathrm{P} / \mathrm{D})\end{array}$ & $\begin{array}{c}\text { HH severity } \\
(\mathrm{X} / \mathrm{XX})\end{array}$ & Preference & $\begin{array}{l}\text { Wanted to } \\
\text { continue** }\end{array}$ \\
\hline 1 & M & $\mathrm{C} 4$ & $P$ & $\mathrm{XX}$ & DP & + \\
\hline 2 & $\mathrm{~F}$ & C6 & $P$ & $\mathrm{XX}$ & None & - \\
\hline 3 & $\mathrm{~F}$ & C6 & $P$ & $\mathrm{XX}$ & None & - \\
\hline 4 & M & C6 & $\mathrm{P}$ & $\mathrm{X}$ & Drop out & - \\
\hline 5 & $\mathrm{~F}$ & C6 & $P$ & $X X$ & DP & + \\
\hline 6 & $\mathrm{~F}$ & C6 & $\mathbf{P}$ & $\mathrm{X}$ & DP & + \\
\hline 7 & $M$ & C6 & $\mathbf{P}$ & $X X$ & DP & + \\
\hline 8 & M & C7 & $\mathbf{P}$ & XX & Drop out & - \\
\hline 9 & $\mathrm{~F}$ & Th3 & $\mathrm{P}$ & $\mathrm{X}$ & DP & + \\
\hline 10 & $M$ & Th4 & $P$ & $\mathrm{X}$ & DP & + \\
\hline 11 & M & Th4 & $\mathrm{D}$ & $X$ & Placebo & - \\
\hline 12 & M & Th4 & $P$ & $\mathrm{X}$ & Drop out & - \\
\hline 13 & M & Th5 & D & $\mathrm{X}$ & DP & + \\
\hline 14 & M & Th5 & $\mathrm{P}^{*}+\mathrm{D}^{*}$ & $X X$ & Drop out & - \\
\hline 15 & M & Th6 & $\mathrm{P}^{*}+\mathrm{D}^{*}$ & $\mathrm{X}$ & Drop out & - \\
\hline 16 & $\mathrm{M}$ & Th7 & D & $\mathrm{XX}$ & Placebo & + \\
\hline 17 & M & Th8 & $\mathrm{D}$ & $\mathrm{X}$ & Placebo & + \\
\hline 18 & M & Th8 & D & $\mathrm{X}$ & None & - \\
\hline 19 & M & Th9 & D & $X X$ & None & - \\
\hline 20 & M & Th9 & $\mathrm{P}^{*}+\mathrm{D}$ & $\mathrm{X}$ & DP & - \\
\hline 21 & M & Th10 & $\mathrm{P}^{*}$ & $X$ & Placebo & - \\
\hline 22 & M & Th11 & $\mathrm{P}^{*}$ & $\mathrm{X}$ & Placebo & + \\
\hline 23 & M & L1 & $\mathrm{P}^{*}$ & $X X$ & Placebo & - \\
\hline 24 & M & L3 & $\mathrm{P}^{*}+\mathrm{D}$ & $\mathrm{X}$ & Drop out & - \\
\hline 25 & M & L4 & D & $X$ & DP & + \\
\hline
\end{tabular}

M: male, F: female, P: proximal to the level of the lesion, D: distal to the level of the lesion $\left({ }^{*}\right.$ indicates sweating less well defined $-\mathrm{cf}$ the text), $\mathrm{X}$ : annoying, $\mathrm{XX}$ : very annoying, ${ }^{* *}$ wanted to continue the preferred treatment.

level most often mentioned in which autonomic hyperreflexia has been seen), the corresponding interval was $40-97 \%$. The possibility of an effect seems to be better in patients with a high SCI (see Fig 1). Eight patients who preferred DP and 3 patients who preferred placebo wanted to continue treatment. This means that a net gain of 5 patients $(8-3)$, ie $26 \%$ of those completing the study, was obtained with the active drug. Analysis of variance of the median visual analogue scale values showed a trend $(p=0.086-0.140)$ towards an effect of DP on sweating during the day.

Adverse effects were recorded in 17 of the 25 patients included and in 12 of the 19 patients who completed the study. In the latter, adverse effects during DP treatment were experienced by 10 patients: lethargy, nausea, dyspepsia, dry mouth, dizziness, or difficulty in emptying bladder. There was a trend $(p=0.075)$ to an increased frequency of adverse effects during active therapy. All drop outs due to adverse effects occurred during DP administration ( $p=0.056)$.

Eighteen of the 19 patients completing the study received other medical treatment, with an average number of 2.2 medicaments per patient. The medicaments given were: muscle relaxants $(\mathrm{n}=8)$, anticonvulsants $(\mathrm{n}=1)$, tricyclic antidepressants $(\mathrm{n}=1)$, sedatives $(n=4)$, alpha-adrenoceptor antagonists $(\mathrm{n}=1)$, bladder muscle relaxants $(\mathrm{n}=1), \quad$ antibiotics $(\mathrm{n}=4)$, diuretics $(n=2)$, urinary acidifying drugs $(n=4)$, laxatives $(n=7)$, and analgesics $(n=2)$. Some patients received 2 medicaments from the same pharmacological group. 


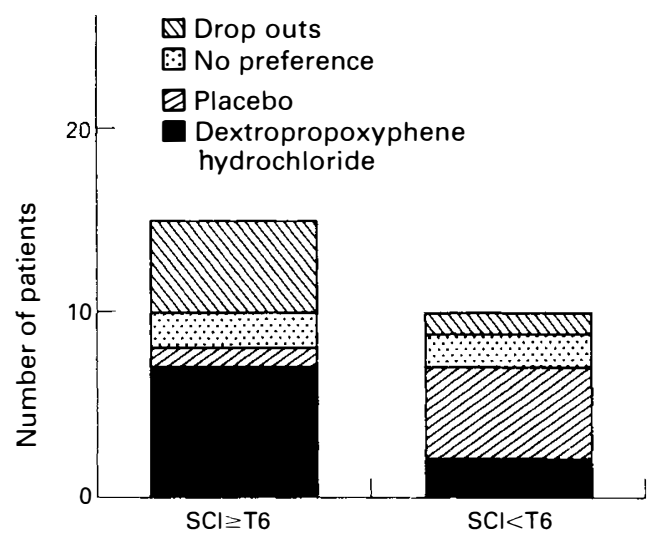

Figure 1 Patient preferences in relation to the level of the lesion. The $95 \%$ confidence interval for the true proportion of patients who preferred dextropropoxyphene hydrochloride, given that the patient had a preference, was for all the patients $32-84 \%$. Considering only the patients with a level of the lesion above or at T6 the corresponding interval was $40-97 \%$.

During the trial period 5 patients were started on antibiotic therapy. Whether infections had any influence on preferences could not be judged.

\section{Discussion}

The prevalence of $\mathrm{HH}$ in SCI patients has not been investigated before to our knowledge. The frequency observed in the questionnaire study seems rather high, as $\mathrm{HH}$ was reported by $29 \%$ of the SCI patients, although only $14.6 \%$ found it annoying and had no contributing somatic cause. Patients with cervical lesions seem to have the most annoying sweating, which is consistent with previous findings. ${ }^{6}$

Previously no controlled study had been performed for the treatment of annoying $\mathrm{HH}$ in SCI. The effect of DP in our study did not reach statistical significance but showed a trend towards effects, especially in patients with SCI at higher lesion levels. A statistically significant effect is probably difficult to achieve in this trial because of the limited number of patients included; the drop out frequency of $20 \%$; and the relatively high frequency of other somatic problems (ie frequent infections, pressure sores, bladder or bowel emptying problems) which probably results in a changing sweating pattern.

Considering that sweating can be very annoying and socially invalidating, that there are only few therapeutic possibilities for treatment, and that no serious adverse effects of DP was reported, we suggest that DP could be tried in individual cases of $\mathrm{HH}$, especially in patients with SCI at or above T6 level.

The pathophysiological explanation for $\mathrm{HH}$, which is most probably the same as reflex sweating, ${ }^{1,7-10}$ is still partially obscure. Changes in the autonomic nervous system and in sweating and temperature regulation in SCI have been the subject of several investigations, ${ }^{1,6-9,11-14}$ and sweating has been found to represent an outstanding component of the symptoms of the spinal mass-reflex. ${ }^{8,14}$ In most cases reflex sweating or $\mathrm{HH}$ is probably mediated through the same mechanisms as autonomic hyperreflexia. ${ }^{1,7}$

In patients with SCI the afferent impulses from below the level of the lesion enter the spinal cord via sympathetic, parasympathetic, and somatosensory nerves through the dorsal nerve root and ascend along the spinothalamic tracts or the dorsal columns. At segmental levels up to the level of the lesion the impulses may cause a spinal mass-reflex, which has also been called autonomic hyperreflexia (or autonomic dysreflexia). ${ }^{6}$ The autonomic hyperreflexia/ dysreflexia has been suggested to be the result of sprouting of ascending fibres, forming abnormal synapses in the intermediolateral grey columns and resulting in a mass discharge of sympathetic neurons from peripheral stimuli. ${ }^{6,15,16}$ Eventually this sympathetic outflow below the level of the lesion results in signs of sympathetic overactivity, such as sweating, pilomotor spasm, and vasoconstriction in the splanchnic vascular bed, the skin, and the legs. Due to the lack of supraspinal inhibition the vasoconstriction persists, especially in patients with complete isolation of the sympathetic splanchnic outflow, as is the case for patients with cervical and high thoracic cord lesions (lesion level above T4). This can result in autonomic hyperreflexia with hypertension, bradycardia, and vasodilata- 
tion proximal to the level of the lesion..$^{2,7,9,17}$ The lowest level most often mentioned in which autonomic hyperreflexia has been seen is T6, ${ }^{1,6}$ a level which is above most of the splanchnic sympathetic outflow, but even at levels down to T11 cases have been reported. ${ }^{16-18}$

The theoretical explanation for autonomic hyperreflexia is supported by observations ${ }^{8}$ of SCI patients in which bladder distention could elicit the characteristic changes in the autonomic nervous system. Sweating was seen as an outstanding feature induced by bladder distension. Almost any afferent impulse of visceral, cutaneous, or proprioceptive origin has been shown to provoke these changes, ${ }^{14}$ and the bladder and rectum have been found to be especially reflexogenic. ${ }^{19}$ Furthermore, List and Pimenta $^{17}$ showed that bladder distension induced sweating could be abolished by regional block of the splanchnic nerves.

Characteristic sweating patterns were described by Head and Riddoch ${ }^{14}$ and were found to be dependent on the level of the lesion. Generally the sweating was most prominent in the most proximal parts, and tapered downward. Patients with cervical lesions had sweating in the head, neck, and arms. Sweating was seen only in the face and neck among patients with lesion levels above T3 level, and extended downwards below the level of the lesion. In patients with lesion levels below or at T9 level sweating was seen only distally to the level of the lesion.

The reasons for the sweating patterns seen in SCI patients are complex and partially unknown. It is still not completely understood $^{1,20,21}$ why patients with cervical and high thoracic SCI typically report the sweating to be located in the head, neck, and arms, ie the dermatomes above the level of the lesion. It seems that there is a higher degree of sympathetic activity in the proximal parts of the isolated cord, since it has been observed that the sweating in SCI with thoracic lesions is generally most active in the proximal parts just below the level of the lesion. ${ }^{1,17}$ For patients with cervical spinal lesions, the most proximal parts of the isolated spinal cord are the upper thoracic segments, and these segments
(T1-T7) are in turn those from which the eccrine sweat glands in the face, neck, and arms receive their sympathetic innervation, which could explain why patients with a high level of lesion typically sweat above the level. Thermoregulatory mechanisms are not responsible for sweating in cervical SCI, as this implies integrity of the sympathetic connections between the thermoregulatory centre in the hypothalamus and the sympathetic efferent nerve fibres to the eccrine sweat glands which originate from the spinal cord segments between T1-L2.,17 Circulating catecholamines have in some reports been elevated during autonomic hyperreflexia ${ }^{6}$ and could induce a weak noncholinergic sweat response in eccrine sweat glands. ${ }^{22}$

In SCI patients with mid and lower thoracic lesion levels, sweating is often seen below the level and tapers downwards; this is explained by the same mechanism as for the cervical SCI. Sweating above the level of the lesion in thoracic SCI patients could be explained by normal thermoregulatory mechanisms ${ }^{23,24}$ activated either by a rise in the core temperature due to pilomotor spasms and vasoconstriction, or by circulating catecholamines. ${ }^{22}$

$\mathrm{HH}$ in patients with lumbar lesions is probably rare, but has been reported by Guttmann in a patient with conus/cauda equina syndrome. ${ }^{1}$

The sweating pattern observed in our patients with cervical and higher thoracic lesion levels (Table II) is consistent with the observations by Head and Riddoch. ${ }^{14}$ Furthermore, our patients reported that sweating could also be induced by the stimuli which typically elicit autonomic hyperreflexia. It is our impression that $\mathrm{HH}$ in most of our patients (especially those with cervical and higher thoracic SCI) is elicited by the same mechanisms as the autonomic hyperreflexia. ${ }^{6}$ Therefore, before starting treatment for annoying $\mathrm{HH}$, one should exclude treatable causes such as distension (constipation or urinary retention) or inflammation of visceral organs, infections, pressure sores, ingrown toe nails, etc.

Other rarer causes of $\mathrm{HH}$ have been reported and also need to be considered, ie post traumatic syringomyelia, ${ }^{25}$ orthostatic 
hypotension, ${ }^{26}$ and dural adhesions to the spinal cord. ${ }^{10,27}$

It would be rational to use medicaments known to affect the mechanisms for reflex sweating and autonomic hyperreflexia. Previously, treatment of $\mathrm{HH}$ has been reported in uncontrolled trials, or in single cases with alpha-adrenoceptor antagonists, for instance phenoxybenzamine ${ }^{28}$ cholinergic blocking drugs such as methantheline,${ }^{25}$ and most recently hyoscine in a patch formula- tion..$^{29}$

In view of the ill understood neurophysiological mechanisms for $\mathrm{HH}$, none of the above mentioned drugs is theoretically more suitable to use than any other. The main point in medical treatment of $\mathrm{HH}$ at this stage is to achieve a maximal therapeutic effect with minimal adverse effects, and we suggest that DP is worthwhile trying especially in patients with spinal cord injury above the level of T6.

\section{References}

1 Guttmann L (1973) Spinal Cord Injuries. Comprehensive Management and Research. Blackwell, Oxford: 271-343

2 Tashjian EA, Richter KJ (1985) The value of propoxyphene hydrochloride (Darvon ${ }^{\circledR}$ ) for the treatment of hyperhidrosis in the spinal cord injured patient. An anecdotal experience and case reports. Paraplegia 23: 349-353.

3 Kayaalp SO, McIsaac RJ (1970) Differential blockade and potentiation of transmission in a sympathetic ganglion. J Pharmacol Exp Ther 137: 193-204.

4 Zelis R, Mansour EJ, Capone RJ, Mason DT (1974) The cardiovascular effects of morphine. J Clin Invest 54: $1247-1258$

5 Prescott RJ (1981) The comparison of success rates in cross-over trials in the presence of an order effect. $J$ $R$ Stat Soc (Series C), Appl Stat 30(1): 9-19.

6 Kewalramani LS (1980) Autonomic dysreflexia in traumatic myelopathy. Am J Phys Med 59(1): 1-21.

7 Fast A (1977) Reflex sweating in patients with spinal cord injury: a review. Arch Phys Med Rehabil 10: 435-437.

8 Guttmann L, Whitteridge D (1947) Effects of bladder distension on autonomic mechanisms after spinal cord injuries. Brain 70: 361-404.

9 Yasuda K, Ito H, Hama T (1978) Management of autonomic hyperreflexia of high spinal cord lesions in acute and recovery stage. Nippon Hinyokiko Gakkai Zasshi 69(12): 1543-1551.

10 Johnson RH (1974) Sweating. Contemp Neurol (series 11): 179-198.

11 Guttmann L (1940) Topographic studies of disturbances of sweat secretion after complete lesion of peripheral nerves. J Neurol Psychiatry 3: 197-210.

12 Johnson RH (1971) Temperature regulation in paraplegia. Paraplegia 9: 135-145.

13 Normell LA (1974) Distribution of impaired cutaneous vasomotor and sudomotor function in paraplegic man. Scand Clin Lab Invest 33 (suppl 138): 25-41.

14 Head H, Riddoch G (1917) The autonomic bladder, excessive sweating, and some other reflex conditions, in gross injuries of the spinal cord. Brain 40: 188-263.

15 Johnson B, Pallares V, Thomason R, Sadove MS (1975) Autonomic hyperreflexia: a review. Mil Med 140: 345-49.

16 Kneisley LW (1977) Hyperhidrosis in paraplegia. Arch Neurol 34: 536-539.

17 List CF, Pimenta AD (1944) Sweat secretion in man. Arch Neurol Psychiatry 51(6): 501-507.

18 Leivy DM (1968) Failure of lumbar sympathectomy in the relief of hyperhidrosis. J Neurosurg 29(1): 65-69.

19 Bors E, French JD (1952) Management of paroxysmal hypertension following injuries to cervical and thoracic segments of spinal cord. Arch Surg 64: 803-12.

20 Kurnick NB (1956) Autonomic hyperreflexia and its control in patients with spinal cord lesions. Ann Intern Med 44: 678-86.

21 Scheibert CD (1955) Studies on the sacral reflex arc in paraplegia. J Neurosurg 12: 468-474.

22 Allen JA, Roddie IC (1972) The role of circulating catecholamines in sweat production in man. J Physiol 227: 801-814.

23 Huckaba C, Frewin DB, Downey JA, Tam HS, Darling RC, Cheh HY (1976) Sweating responses of normal, paraplegic and anhidrotic subjects. Arch Phys Med Rehabil 57: 268-274.

24 Downey JA, Huckaba CE, Kelley PS, Tam HS, Darling RC, Cheh HY (1976) Sweating responses to central and peripheral heating in spinal man. J Appl Physiol 40(5): 701-706.

25 Stanworth PA (1982) The significance of hyperhidrosis in patients with post-traumatic syringomyelia. Paraplegia 20: 282-287.

26 Khurana RK 1987 Orthostatic hypotension-induced autonomic dysreflexia. Neurology 37: 1221-1224.

27 Ottomo M, Heimburger RF (1980) Alternating Horners syndrome and hyperhidrosis due to dural adhesions following cervical spinal cord injury. J Neurosurg 53(1): 97-100. 
28 Shessel FS, Carrion HM, Politano VA (1978) Phenoxybenzamine and sweating in the spinal cord injury patients. J Urol 120(1): 60-61.

29 Staas WE, Nemunaitis G (1989) Management of reflex sweating in spinal cord injured patients. Arch Phys Med Rehabil 70: 544-546. 